\title{
Direct Evidence of a Transition from Triplet Excitons to Hybrid Light-Matter States via Triplet-Triplet Annihilation
}

\author{
Chen Ye ${ }^{\mathrm{a}}$, Suman Mallick ${ }^{\mathrm{a}}$, Markus Kowalewski ${ }^{\mathrm{b}}$ and Karl Börjesson ${ }^{\mathrm{a} *}$
}

\begin{abstract}
Strong light-matter coupling generates hybrid states that inherit properties of both light and matter, effectively allowing the modification of the molecular potential energy landscape. This phenomenon opens up a plethora of options to manipulate the properties of molecules, with a broad range of applications in photochemistry and photophysics. In this contribution, we design an on-off photon-upconversion system providing a direct evidence of a conversion between molecular states and hybrid light-matter states. The conversion follows the basic principle of spin conservation in triplet-triplet annihilation. Our experiment shows that our method allows for the transformation of an endothermic triplet-triplet annihilation process into a more efficient exothermic one. These results provide the first on-off evidence that energy can relax from non-resonant low energy molecular states directly into hybrid light matter states, and lay the ground work for tunable photon up-conversion devices that modify molecular properties in situ by optical cavities rather than with chemical modifications.
\end{abstract}

\section{Introduction}

The interaction between light and matter contributes to the fundamental movement of the universe, and has been an essential part of scientific research since ancient times. ${ }^{1}$ Photons

[a] Dr. C. Ye, Dr. S. Mallick, Dr. K. Börjesson

Department of Chemistry and Molecular Biology, University of Gothenburg, Kemigården 4, 41296 Gothenburg (Sweden)

[b] Dr. M. Kowalewski Department of Physics, Stockholm University, Albanova University Centre, 10691 Stockholm (Sweden)

E-mail: karl.borjesson@gu.se

Supplementary information (SI) for this article is given via a link at the end of the document. 
interact with the induced dipole of molecular transitions, and when the strength of this interaction is larger than energy dissipation from the system, the strong coupling regime is reached. ${ }^{1-3}$ This phenomenon leads to the generation of light-matter hybrid states, called polaritons (or dressed states; Scheme 1). Polaritons are quasi particles having both photonic and excitonic contributions, and therefore inherit properties from both. ${ }^{4,5}$ The photonic contribution results in a delocalized character and the excitonic contribution enables material interactions. ${ }^{6,7}$ The use of optical cavities instead of laser generated fields allows to realize these modifications by means of passive structures rather than active optical devices. Researchers have recently utilized these unique properties to accomplish entirely new developments in optics, ${ }^{8}$ electronics, ${ }^{9}$ catalysis, ${ }^{10}$ and quantum devices ${ }^{11}$.

In condensed phase, strong light-matter coupling was first achieved with inorganic semiconductors, which normally forms loosely bounded Wannier-Mott excitons. ${ }^{12,13}$ These inorganic Wannier-Mott excitons have small exciton binding energies and large wave function overlap, and their strong light-matter coupling properties have been intensively studied. ${ }^{3,14}$ Compared to inorganic semiconductors, organic molecules normally form Frenkel excitons with large transition dipole moments and consequently increased light-matter coupling, and has furthermore access to a richer variety of photochemical and photophysical processes. Organic strong light-matter coupling therefore offer new opportunities, and is currently generating a large research interest. ${ }^{15,16}$ Recent advances probe the transition between polaritonic and molecular states by observing differences in rates, ${ }^{17}$ including FRET energy transfer, ${ }^{18,19}$ reverse intersystem crossing, ${ }^{20,21}$ and triplet fusion/singlet fission. ${ }^{22,23}$

Among these types of excited states transitions, triplet-triplet annihilation (TTA) can efficiently convert low energy photons to high energy ones, ${ }^{24,25}$ and therefore offers a generic method 
of boosting the maximal efficiency of a single junction photovoltaic device from $32 \%$ to $51 \% .^{26,27}$ TTA occurs from the close interaction between two excited triplet states (triplet pair), when their energy level configuration meets a certain condition $\left(2 E_{T 1}>E_{\mathrm{S} 1}\right){ }^{26,27}$ Organic synthesis is an effective strategy to tune molecular energy levels for meeting the TTA condition. ${ }^{28}$ However, this strategy is time consuming and available synthesis pathways and the laws of molecular photophysics limit the resulting molecules.

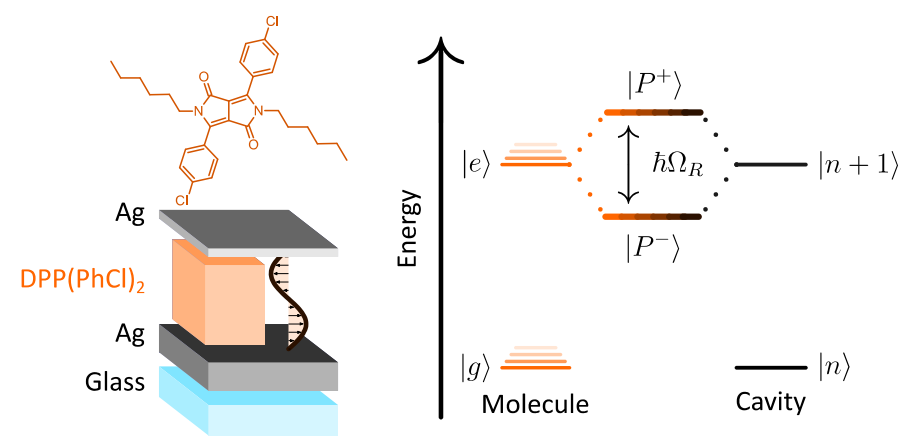

Scheme 1. Schematic illustration of strong light-matter coupling. Molecular structure of $\mathrm{DPP}(\mathrm{PhCl})_{2}$, cavity configuration, and energetic landscape of molecular states under strong light-matter coupling.

Here we demonstrate the conversion of triplet excitons directly to hybrid light-matter states by turning an endothermic TTA process into an exothermic one. We start by showing that it is possible to couple a TTA-unfavored molecule strongly to the vacuum field, and by so doing create cavity polaritons having the correct energetics for exothermic TTA. Using temperature resolved photoluminescence spectroscopy, we continue with showing that TTA is only observed at low temperatures when energy accessible cavity polaritons are present. Modelling of the TTA process is performed using data provided by time-resolved spectroscopy, confirming the view of a new TTA pathway opening up in the strong coupling regime. Finally, we elucidate that the transition from molecular states to hybrid light-matter states via TTA is spin conserved. 


\section{Results and discussion}

\subsection{Molecular photophysics of the annihilator}
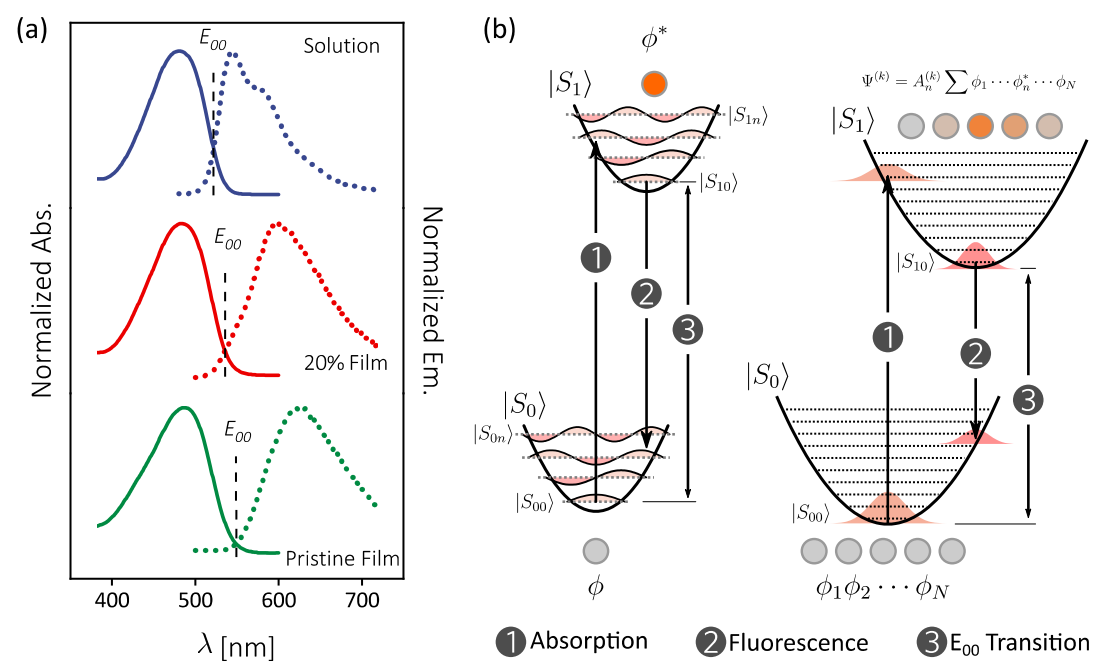

Figure 1. Molecular photophysics from dilute solution to pristine film. (a) Normalized absorption of $10^{-5} \mathrm{M} \mathrm{DPP}(\mathrm{PhCl})_{2}$ in toluene solution, $\operatorname{DPP}(\mathrm{PhCl})_{2} /$ polystyrene film $(\mathrm{w} / \mathrm{w} \%=20 \%)$, and pristine $\mathrm{DPP}(\mathrm{PhCl})_{2}$ film; (b) Energy landscape of independent $\mathrm{DPP}(\mathrm{PhCl})_{2}$ molecules and $\mathrm{DPP}(\mathrm{PhCl})_{2}$ excitons in pristine films.

Aromatic molecules, like anthracene, pyrene, and perylene, are typical TTA annihilators due to their long lived triplet states and the appropriate singlet-triplet energy alignment. The $\mathrm{S}_{1}$ state is generally less distorted than the $T_{1}$ state compared to the ground state, and it is therefore more sensitive to substitution. ${ }^{29}$ Chemists took advantage of this effect, and developed a library of diketopyrrolopyrrole (DPP) annihilators to sweep the singlet energy without perturbing the triplet energy. ${ }^{28}$ We show here that strong coupling can do the same without synthesis. This is achived by coupling 3,6-bis(4-chlorobenzene)-diketopyrrolopyrrole $\left(\mathrm{DPP}(\mathrm{PhCl})_{2}\right)$ strongly to the electromagnetic field. Two times the $\mathrm{T}_{1}$ energy of $\mathrm{DPP}(\mathrm{PhCl})_{2}(1.15$ $\mathrm{eV})$ is slightly lower than its $\mathrm{S}_{1}$ energy $(2.36 \mathrm{eV})$. This energy level alignment therefore makes $\mathrm{DPP}(\mathrm{PhCl})_{2}$ TTA-silent. Figure $1 a$ shows the absorption and emission spectra of $\mathrm{DPP}(\mathrm{PhCl})_{2}$ in dilute solution and in solid state. After excitation to the Franck-Condon state, $\mathrm{DPP}(\mathrm{PhCl})_{2}$ molecules in solution quickly relax to the lowest vibrational level of the singlet excited state. 
In the pristine solid state, Frenkel exciton bands are formed. The exciton band energy tends to be reduced and more continuous as the molecular interaction increases (Figure $1 b$ ). The energy of the Frank-Condon state, as well as the relaxed excited state, were determined in solution and neat films for coming discussion.

\subsection{Entering the strong coupling regime}

The large transition dipole moment of $\operatorname{DPP}(\mathrm{PhCl})_{2}$ enables the collective strong light-matter coupling regime to be reached. The coupling is obtained by placing a triplet sensitizer doped $\operatorname{DPP}(\mathrm{PhCl})_{2}$ thin film within a Fabry-Pérot cavity (Scheme 1, SI 1.3). The cavity consist of a 30 $\mathrm{nm}$ Ag top mirror and a $100 \mathrm{~nm}$ Ag bottom mirror sandwiching the molecular film. The optical cavity confines the electromagnetic field at the optical resonance dictated by the cavity thickness. When the optical resonance matches the molecular transition, the cavity and molecule couples (Scheme 1). We characterized the strong light-matter coupling as a function of cavity resonance tuning. Figure $2 a$ show angle resolved reflectivity of three cavities (Cav1, Cav2, and Cav3) tuned differently over the energy of the molecular exciton (see Figure S2 for Cav4-8). For these cavities, the molecular absorption splits into two polaritonic branches, $\left|\mathrm{P}^{-}\right\rangle$ and $\left|\mathrm{P}^{+}\right\rangle$. Both branches are shifted to higher energies as the incident angle increases (Figure $2 b)$, but $\left|\mathrm{P}^{-}\right\rangle$never crosses the energy of the molecular exciton. The observed anti-crossing is a typical feature of strong light-matter coupling. To extract the coupling strength, polariton energies, and composition, the dispersion of polariton energies were fitted to a JaynesCummings type model (eq. 1):

$$
\left[\begin{array}{cc}
E_{c}(\theta) & \frac{\hbar \Omega}{2} \\
\frac{\hbar \Omega}{2} & E_{\text {ex }}
\end{array}\right]\left[\begin{array}{l}
\alpha \\
\beta
\end{array}\right]=E(\theta)\left[\begin{array}{l}
\alpha \\
\beta
\end{array}\right](\text { eq. } 1)
$$


where $E_{c}$ is the cavity photon energy, which is related to the incident angle $\theta, E_{e x}$ is the exciton energy (the Frank-Condon state), and $\hbar \Omega$ is the Rabi splitting. The in-plane distribution of polariton energies are obtained from the eigenvalues of the Hamiltonian. The Hopfield coefficients, $|\alpha|^{2}$ and $|\beta|^{2}$, represent the fractional excitonic and photonic contributions to the corresponding polaritons. ${ }^{30}$ The collective Rabi splitting ( $0.44 \mathrm{eV}$ for all) is larger than the full width at half maximum (FWHM) of the molecular transition $(0.42 \mathrm{eV})$, indicating that the coupling strength is larger than dissipation. Furthermore, the relative coupling strength is 0.18 (defined as the Rabi splitting over the excitonic energy), supporting the entrance to the strong coupling regime.

(a)
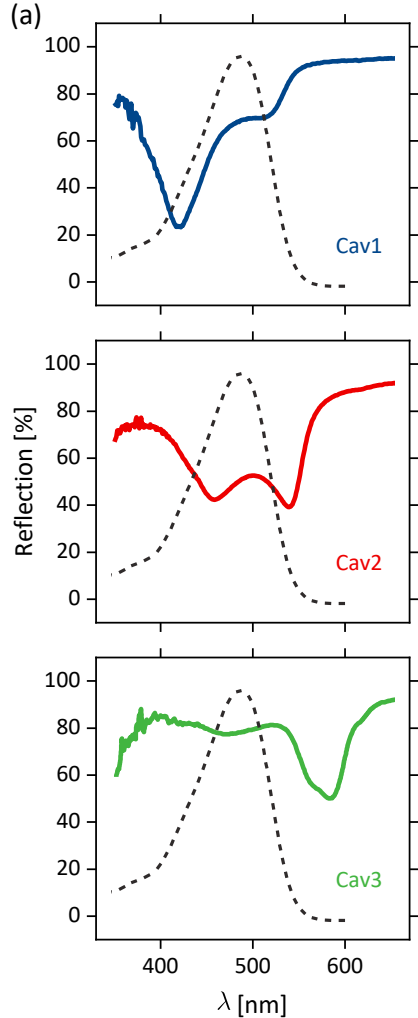

(b)
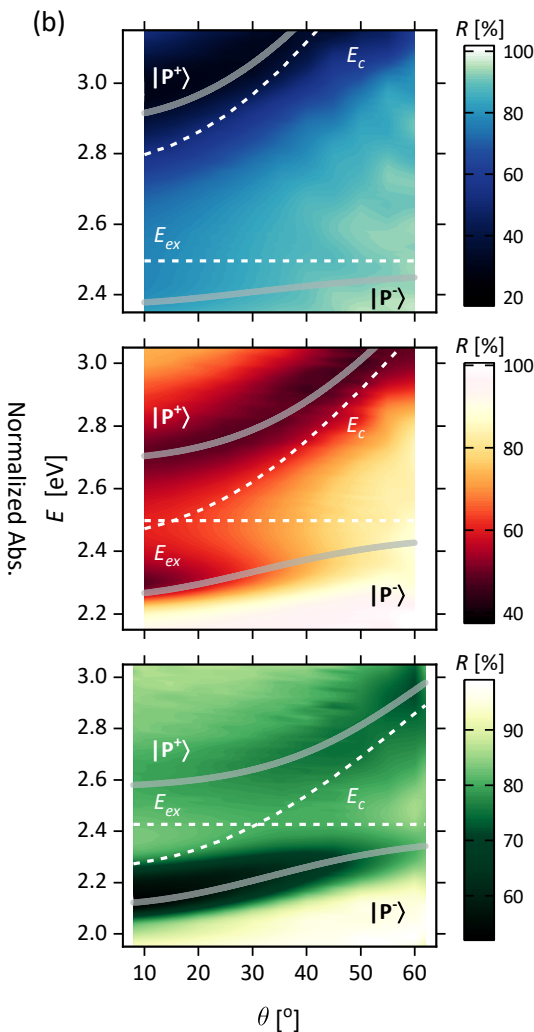

(c)
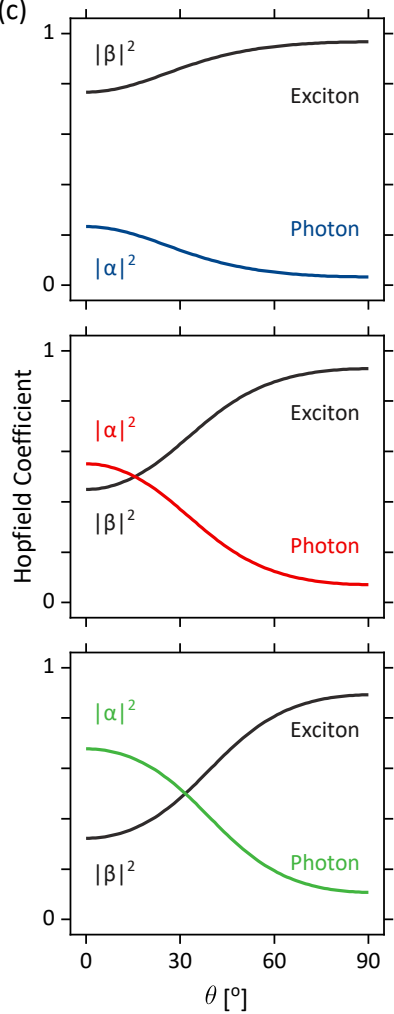

Figure 2. Characterizing the strong light-matter interaction. (a) Comparison of the reflectance spectra of sensitizer/DPP $(\mathrm{PhCl})_{2}$ cavity at different tunings with the molecular absorption of the sensitizer/DPP $(\mathrm{PhCl})_{2}$ bare film; (b) Angle-resolved reflectivity of sensitizer/DPP $(\mathrm{PhCl})_{2}$ cavities at different tuning together with a fit to a coupled oscillator model; (c) Hopfield coefficient of $\left|\mathrm{P}^{-}\right\rangle$as a function of incidence angle. 
Strong light-matter coupling provides a possibility to tune the energy level alignment by splitting the Franck-Condon state into two polariton branches (Scheme 2). As the cavity resonance energy is decreasing, the $\left|\mathrm{P}^{-}\right\rangle$energy decreases below the energy of the relaxed exciton $\left(E_{00}\right)$. Furthermore, when the cavity resonance energy varies, the exitonic and photonic contribution to $\left|\mathrm{P}^{-}\right\rangle$also changes (Figure 2c). By tuning the cavity resonance frequency to lower energies, the energy of $\left|\mathrm{P}^{-}\right\rangle$decreases, but the exciton fractional contribution to $\left|\mathrm{P}^{-}\right\rangle$also reduces. As we will show later, with a too low excitonic contribution to $\left|\mathrm{P}^{-}\right\rangle$, the connection between materials centered and polaritonic states diminish, limiting the practical tuning range.

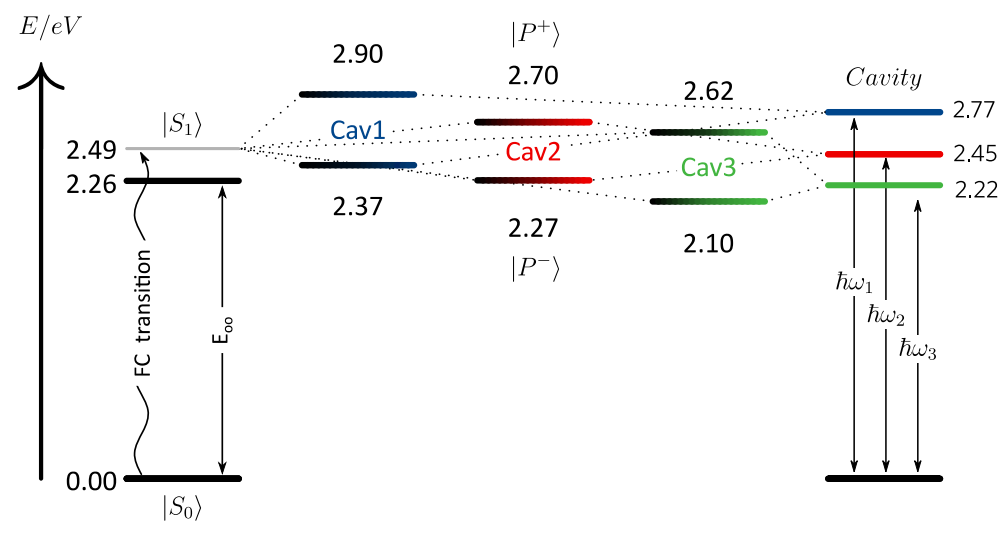

Scheme 2. Energy configuration of the system. Energy configurations of the $\mathrm{DPP}(\mathrm{PhCl})_{2}$ molecule in bare film and cavities at zero incident degree with different tuning.

\subsection{Photon upconversion and polariton emission}

We have so far demonstrated that the singlet excited state of $\operatorname{DPP}(\mathrm{PhCl})_{2}$ can be strongly coupled to the vacuum electromagnetic field. We will here show that the change in energy level alignment by the formation of polaritonic states has a profound effect on the system's ability to perform TTA, giving large evidence for a direct pathway from the excitonic triplet pair to $\left|\mathrm{P}^{-}\right\rangle$. As a triplet sensitizer, we choose platinum tetra-benzo-tetra-phenyl-porphyrin (PtTBTP) at a doping concentration of $1 \%$ in otherwise pristine $\operatorname{DPP}(\mathrm{PhCl})_{2}$ films $(\mathrm{SI} 2.2)$. The 
high intersystem crossing efficiency and appropriate triplet energy ( $1.61 \mathrm{eV})$ makes PtTBTP a suitable triplet sensitizer for $\operatorname{DPP}(\mathrm{PhCl})_{2}$. The small amount of doped sensitizer is not coupled to the cavity due to the mismatch in resonance frequency. Pun et al. found that triplet $\operatorname{DPP}(\mathrm{PhCl})_{2}$ in solution is unable to undergo efficient TTA due to the endothermic nature of the process $\left(\Delta E=E_{\mathrm{S} 1}-2 E_{\mathrm{T} 1}=60 \mathrm{meV}\right){ }^{28}$ Thus, the triplet $\mathrm{DPP}(\mathrm{PhCl})_{2}$ relaxes through monomolecular intrinsic decay in solution. We examined the TTA behavior of PtTBTP/DPP $(\mathrm{PhCl})_{2}, \mathrm{PtTBTP}$, and $\operatorname{DPP}(\mathrm{PhCl})_{2}$ films under $\mathrm{N}_{2}$ atmosphere using a pulsed excitation source $\left(\lambda_{e x}=613 \mathrm{~nm}\right)$. The phosphorescence lifetime of PtTBTP decreases from 17.6 $\mu \mathrm{s}$ in PtTBTP pristine films to $3.1 \mu \mathrm{s}$ in PtTBTP/DPP $(\mathrm{PhCl})_{2}$ films (Figure S4, SI 2.3). The order of magnitude reduced PtTBTP lifetime in the presence of $\mathrm{DPP}(\mathrm{PhCl})_{2}$ indicate an efficient triplettriplet energy transfer from PtTBTP to $\mathrm{DPP}(\mathrm{PhCl})_{2}$. The reduced sensitizer emission in PtTBTP/DPP $(\mathrm{PhCl})_{2}$ films is followed by weak molecular emission from $\mathrm{DPP}(\mathrm{PhCl})_{2}$. The emission exhibits the same spectral envelope as the directly excited pristine $\mathrm{DPP}(\mathrm{PhCl})_{2}$ film $\left(\lambda_{e x}=490 \mathrm{~nm} ;\right.$ Figure $\left.3 a\right)$. The time-resolved emission shows that the emission lasts for a few hundred microseconds, which is about five orders of magnitude longer than the lifetime of fluorescence (Figure S5). Furthermore, we observed no emission from a pristine $\mathrm{DPP}(\mathrm{PhCl})_{2}$ film when exciting at the absorption maximum of PtTBTP $\left(\lambda_{e x}=613 \mathrm{~nm}\right.$; Figure S6). We then infer that $\mathrm{DPP}(\mathrm{PhCl})_{2}$ is able to perform TTA in the solid state under pulsed excitation conditions. Although the $E_{00}$ energy is $100 \mathrm{meV}$ lower for $\mathrm{DPP}(\mathrm{PhCl})_{2}$ in the solid state as compared to dilute solution, the triplet energy might also differ, and the temperature dependence of TTA was therefore investigated to assess if annihilation is endothermic (thermally activated) or exothermic. Figure $3 b$ show that the solid state TTA of $\mathrm{DPP}(\mathrm{PhCl})_{2}$ is strongly dependent on temperature, and the emission becomes undetectable below ca. 130 $\mathrm{K}$. Thus, $\mathrm{DPP}(\mathrm{PhCl})_{2}$ triplet-triplet annihilation in the solid state is endothermic. 

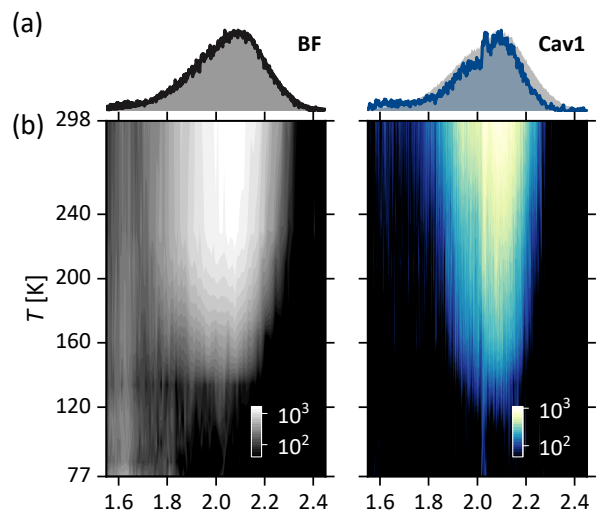

$\begin{array}{lllll}1.6 & 1.8 & 2.0 & 2.2 & 2.4\end{array}$

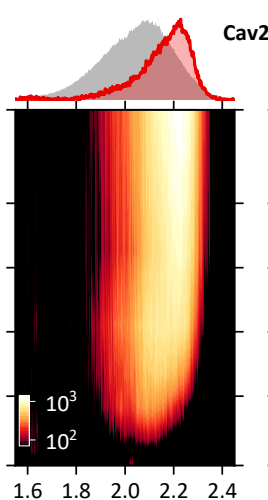

$E[\mathrm{eV}]$
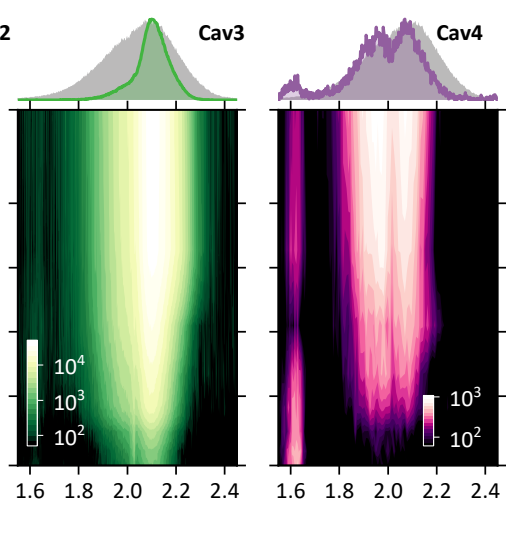

(c)

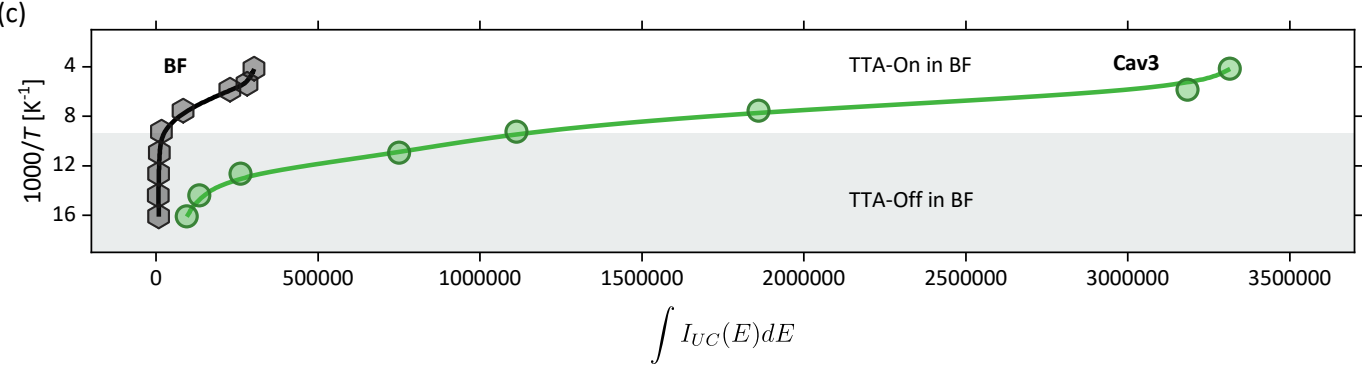

Figure 3. Temperature dependence of TTA-UC emission. (a) Normalized prompt emission from bare film (grey shaded area) and sensitized TTA emission (solid lines) at room temperature; (b) Temperature dependent TTA emission of sensitizer/DPP $(\mathrm{PhCl})_{2}$ bare film and cavities $\left(\lambda_{e x}=613 \mathrm{~nm}, l_{\text {ex }}=0.19-0.23\right.$ $\mathrm{mJ} /$ pulse) after $1 \mu$ s delay; (c) Integrated temperature dependent TTA emission of bare film and Cav3.

The temperature dependent TTA behavior of PtTBTP/DPP $(\mathrm{PhCl})_{2}$ films was examined in the strong coupling regime using the same setup as for pristine films. Four cavities (cav1-4), all having different thicknesses and therefore having different energy level alignment and material contribution to $\left|\mathrm{P}^{-}\right\rangle$were investigated. Cav3 shows polariton emission when directly exciting $\operatorname{DPP}(\mathrm{PhCl})_{2}$. This is evident from a dispersive emission envelope resembling the $\left|\mathrm{P}^{-}\right\rangle$ absorption rather than the non-dispersive bare molecular emission (Figure S7, SI 2.5). The lack of polariton emission from Cav1 can be attributed by the higher energy of $\left|\mathrm{P}^{-}\right\rangle$as compared to the vibrationally relaxed excited singlet state $\left(E_{00}\right)$ of $\operatorname{DPP}(\mathrm{PhCl})_{2}$, and for Cav4 the very large detuning prohibits light-matter coupling. The same emission profiles of the four cavities is present when exciting the sensitizer as compared to direct excitation. Cav2\&3 show polariton emission, and Cav1\&4 display only excitonic emission. Furthermore, the oxygen sensitivity and 
excitation intensity dependence indicate that the photoluminescence originates from TTA (SI

\section{6, Figure S8-S9).}

We now turn our attention to the divergent TTA properties in cavities as compared to bare films at different temperatures, as to confirm that TTA can convert triplet excitons directly to light-matter states. At room temperature, upconverted emission from Cav3 is one order of magnitude stronger than for the other cavities and the bare film, despite the fact that the sensitizer inside the cavity receives less excitation light. ${ }^{31}$ Cav3 has the lowest $\left|\mathrm{P}^{-}\right\rangle$energy, and therefore the largest potential for exothermic upconversion (Scheme 2). Furthermore, the $\mid \mathrm{P}^{-}$ > emission from TTA show a different temperature dependence as compared to the bare film (Figure $3 b)$. TTA of triplet $\mathrm{DPP}(\mathrm{PhCl})_{2}$ to $\left|\mathrm{P}^{-}\right\rangle$is efficient in $\mathrm{Cav3}$, even at temperatures from 77 $\mathrm{K}$ to $130 \mathrm{~K}$. This is in contrast to the bare film, where no emission could be detected at these temperatures. This on-off effect shows that TTA can convert triplet excitons to polaritons without going through an intermediate singlet exciton. To further examine cavity detuning on TTA, three additional cavities having small detuning's compared to Cav3 were made. In a $\left|\mathrm{P}^{-}\right\rangle$ energy interval of $0.08 \mathrm{eV}\left(675 \mathrm{~cm}^{-1}\right)$ all showed upconversion intensities at comparable magnitudes to Cav3 (Figure S10). The detuning interval is small compared to electronic driving forces, but large compared to molecular vibrational energies. Therefore, we can exclude vibrational alignment as a reason for the increased upconversion intensity. In summary, the on-off emission between cavities and bare films indicate a direct upconversion pathway from the triplet pair to the polaritonic state.

\subsection{Photon upconversion kinetics}

To further investigate the mechanism of exciton to polariton TTA, time resolved photoluminescence analysis was performed and a corresponding kinetic model was built. TTA 
in the solid state requires the close interaction between two triplet excitons, and thus involves exciton diffusion in the solid matrix. ${ }^{32}$ The TTA kinetics can therefore be described by the following equation (eq. 2):33

$$
\left\{\begin{array}{c}
\frac{\partial \rho}{\partial t}=D \nabla \rho-\gamma_{T T A} \rho^{2}-k_{T} \rho \\
\left.\rho\right|_{t=0}=\rho_{0}
\end{array}\right.
$$

where $\rho$ is the density of triplet excitons, $D$ is the diffusion coefficient of triplet excitons, $\nu_{T T A}$ is the annihilation rate constant in solid state, and $k_{T}$ is the intrinsic decay constant of triplet excitons, $\rho_{0}$ is the initial density of triplet excitons after excitation. TTA in the solid state is a process in diffusion equilibrium. ${ }^{34}$ The Smolochowski theory can therefore be used to simplify equation 2 giving (eq.3-4):

$$
\begin{gathered}
\frac{d \rho}{d t}=-\gamma_{T T A} \rho^{2}-k_{T} \rho \quad(e q .3) \\
I_{D F} \propto \gamma_{T T A} \rho^{2} \quad(e q .4)
\end{gathered}
$$

where $I_{D F}$ is the intensity of delayed fluorescence. ${ }^{35,36}$ Transient absorption of the bare film was recorded to calculate the initial density of triplet $\mathrm{DPP}(\mathrm{PhCl})_{2}$ after laser excitation $(\mathrm{SI}$ 2.7, Figure S11-S13). We then monitored the time evolution of TTA emission of PtTBTP/DPP $(\mathrm{PhCl})_{2}$ films inside (Cav3) and outside (bare film, BF) a cavity at different temperatures and excitation intensities (SI 2.8, Figure S14-S19). From the results of the fit, we determined that triplet excitons have the same intrinsic decay lifetime inside and outside the cavity (Figure 4a). This indicates that the triplet exciton itself is not affected by the vacuum field inside the cavity. This is in accordance with expectations as triplet excitons have a very small transition dipole moment and the cavity isn't tuned to the energy of the triplet state. The exciton to exciton TTA rate constant of $\mathrm{DPP}(\mathrm{PhCl})_{2}$ follows an Arrhenius type behavior with temperature (Figure 
$4 b)$. By that we confirm that TTA in PtTBTP/DPP $(\mathrm{PhCl})_{2}$ films is an endothermic process, and calculated the energy gap ( $\left.\Delta E=E_{\mathrm{S} 1}-2 E_{\mathrm{T} 1}\right)$ to $57 \mathrm{meV}$. The value is close to that in solution (60 meV). ${ }^{28}$ To summarize, the rate of exciton to exciton TTA of $\operatorname{DPP}(\mathrm{PhCl})_{2}$ in solid state is annihilation limited due to the endothermicity of the reaction.
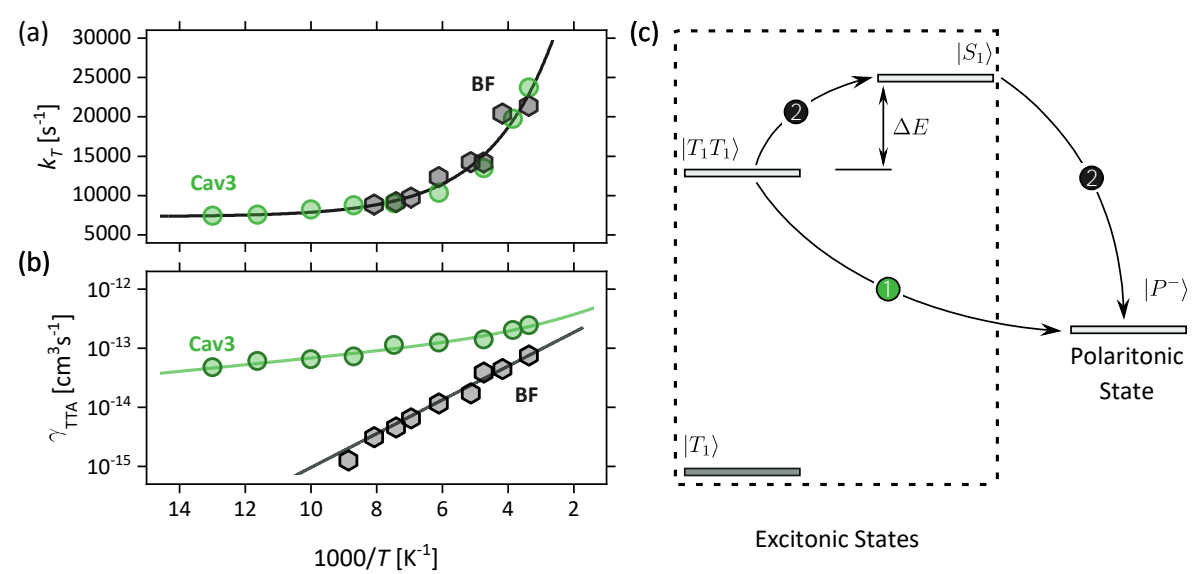

Figure 4. Dynamics of TTA-UC emission. (a) Calculated intrinsic decay rate constant of DPP $(\mathrm{PhCl})_{2}$ inside (Cav3) and outside (BF) a cavity; (b) Calculated TTA rate constant of DPP( $\mathrm{PhCl})_{2}$ inside (Cav3) and outside (BF) a cavity; (c) Schematic illustration of the exciton to polariton energy pathway.

By coupling the exciton to the electromagnetic field, hybrid light-matter states having the ability for exothermic TTA are created. The TTA rate constant in Cav3 is much larger than that in the bare film, especially at low temperatures (Figure $4 b$ ). To accurately fit the temperature dependence of TTA in Cav3, two different TTA channels are needed (Figure 4c). The first term comes from direct exciton to polariton TTA and is required to explain TTA at low temperatures. We assume this transformation is diffusion controlled. This because the rate constant of annihilation is less dependent on temperature as compared the one of the bare film. The second term is exciton to exciton TTA followed by relaxation to $\left|\mathrm{P}^{-}\right\rangle$. This term is needed to explain a slight increase in temperature dependence of the TTA rate constant at higher temperatures. To summarize, a model including direct annihilation from triplet excitons to polaritons is needed to explain our kinetic data. At low temperatures upconversion happens 
only directly to the low energy polaritonic state, whereas at higher energies, excitonic upconversion followed by energy relaxation to $\left|\mathrm{P}^{-}\right\rangle$contribute slightly (Figure S20).

\subsection{Spin statistics of exciton to polariton TTA}

As demonstrated in the previous sections, two triplet excitons can annihilate directly to polaritonic states. We here turn our attention to the spin of the polaritonic state, and on the possibility of a spin conserved excitonic to polaritonic TTA process. Exciton to exciton TTA obeys the basic principles of spin conservation, which enables a high TTA rate constant (SI 2.9, Scheme S2). ${ }^{37}$ Here we assume that the sample is free of an external magnetic field, the observed product states stem from the singlet state, and that spin-orbital coupling in the product is slow compared to fluorescence and polariton decay. Under these assumptions the total angular momentum $\boldsymbol{J}$ must be conserved:

$$
\boldsymbol{J}=\boldsymbol{S}_{\boldsymbol{p}}+\boldsymbol{L}+\boldsymbol{S}_{\boldsymbol{m}}(\text { eq.5) }
$$

where $S_{p}$ is the spin (or helicity) of the photons in the cavity, $L$ is the molecular orbital angular momentum of the product state, and $S_{m}$ is the spin angular momentum of the molecule. Since we have already assumed that the system is in a singlet state and $\boldsymbol{S}_{\boldsymbol{m}}$ is preserved (Scheme S2), eq. 5 reduces to:

$$
\boldsymbol{J}=\boldsymbol{S}_{\boldsymbol{p}}+\boldsymbol{L}(\text { eq. } 6)
$$

In our experiment the cavity couples to the $S_{0}$ to $S_{1}$ transition forming the polariton states and thus corresponds to an exchange of a single cavity photon with the molecular ensemble. The polariton states can be written in terms of Fock-states and molecular electronic states:

$$
\begin{aligned}
& \left.\left|P^{+}\right\rangle=\alpha\left|S_{0}, 1\right\rangle+\beta\left|S_{1}, 0\right\rangle \quad \text { eq. } 7\right) \\
& \left|P^{-}\right\rangle=\alpha\left|S_{0}, 1\right\rangle-\beta\left|S_{1}, 0\right\rangle \quad \text { eq. 8) }
\end{aligned}
$$


where $|0\rangle$ and $|1\rangle$ are the zero and one photon Fock states of the cavity respectively. Here we can assume that the vacuum state $|0\rangle$ of the cavity contributes no spin $\left(\left|\boldsymbol{S}_{p}\right|=0\right)$, and the one photon state $|1\rangle$ contributes one spin quanta $\left(\left|S_{p}\right|=1\right)$. A closed shell molecular ground state has $L=0$ and a dipole allowed, bright $\mathrm{S}_{1}$ state has $|L|=1\left(\pi-\pi^{*}\right.$ transition for $\left.\operatorname{DPP}(\operatorname{PhCl})_{2}\right)$. Thus both polariton states must have $|J|=1$ to conserve the total angular momentum. In conclusion, both polariton states are directly accessible in the TTA process, and the total molecular spin is preserved. The familiar dipole selection rules hold. The spin statistics of TTA is thus influenced only by the energetics of the polaritons (as in the cavity-free case), which adjusts the different spin states relative to each other in terms of energy.

\section{Conclusions}

We have demonstrated that hybrid light matter states can be populated directly from triplet states via triplet-triplet annihilation. By doing so, we turn a TTA-unfavored molecule into a TTA-favored one (a TTA annihilator). Our on-off results is an unambiguous evidence that triplet excitons can be converted directly into polaritons, without any intermediate steps. Thus constituting the first evidence not based on differences in rates, that energy can relax from non-resonant low energy molecular states directly into hybrid light matter states. The polariton states have a lower energy than two times the triplet exciton energy, thus fundamentally changing the energetics of the TTA reaction. The measurements of the temperature dependent TTA kinetics revealed that the triplet exciton itself is not coupled to the optical cavity. However, two triplet excitons can directly convert into exciton-polaritons by TTA. We further demonstrated that exciton to polariton TTA is a spin conserved process, which provides an explanation for the high efficiency of exciton to polariton TTA. The easy tunability of optical nano-cavities makes strong coupling especially versatile for modifying 
fundamental properties of molecular based systems. We anticipate that the principle presented in this paper may become more widely applied in modern molecular science as a tool for building easily tunable systems, and used to improve organic solar cells and other photoelectric devices

\section{Methods}

Cavity preparation. The Fabry-Pérot cavities were built on $1 \mathrm{~cm} \times 1 \mathrm{~cm}$ clean glass substrates. The $100 \mathrm{~nm}$ bottom mirrors were deposited by vacuum sputtering (HEX, Korvus Technologies). The molecular films were then deposited on the bottom mirrors by spin-coating (Laurell Technologies WS6). The $30 \mathrm{~nm}$ top mirrors were deposited on the top of molecular layer by vacuum sputtering.

Modelling the angle-resolved reflectivity. The angle-resolved reflectivity of cavities were fitted by a coupled oscillator model (eq.1). The energy dispersion of cavities $\left(E_{c}\right)$ are given by:

$$
E_{c}(\theta)=\frac{E_{c}(0)}{\sqrt{1-\left(\frac{\sin \theta}{n_{\text {eff }}}\right)^{2}}}(\text { eq. } 9)
$$

where $\theta$ is the incident angle, and $\eta_{\text {eff }}$ is the refractive index inside the cavity. The energy dispersion of the polariton states were then obtained from the eigenvectors of the Hamiltonian in eq. 1.

Steady state absorption, reflection and emission spectroscopy. Absorption spectra for solution samples and films were recorded on a PerkinElmer LAMBDA 950 spectrometer. Angle resolved reflectivity spectra of cavities were recorded on a PerkinElmer LAMBDA 950 spectrometer with a universal reflectance accessory. 
Steady state emission of solution and films were measured on an Edinburgh Instruments FLS 1000 spectrofluorometer with a Xenon lamp as excitation source. Angular resolved emission spectra of cavities were measured on the same instrument using a fiber induced angleresolved platform.

TTA photon upconversion emission. TTA photon upconversion experiments of bare films and cavities were carried out on an Edinburgh Instruments LP 980 transient absorption spectrometer. A Spectra-Physics Nd:YAG $532 \mathrm{~nm}$ laser (pulse width $7 \mathrm{~ns}$ ) coupled to a SpectraPhysics primoscan optical parametric oscillator (OPO) was used as excitation source. An Andor iSatr 334 fast gated intensified CCD was used as the detector. All spectra were recorded with a 4 ms gate width to cover the whole TTA signal and a 500 ns gate delay to avoid scattering and prompt emission. All samples were placed inside an Oxford Instrument Optistat DN-V cryostat with $\mathrm{N}_{2}$ atmosphere. The temperature of the samples were controlled by a MercuryiTC temperature controller.

Time resolved photoluminescence spectroscopy. Time resolved photoluminescence decay of the bare film and cavities were recorded in an $\mathrm{N}_{2}$ atmosphere provided by a cryostat on an Edinburgh Instruments FLS 1000 spectrofluorometer in Multi-Channel Scaling (MCS) mode. A Spectra-Physics Nd:YAG laser (pulse width 7 ns) coupled to a Spectra-Physics primoscan optical parametric oscillator (OPO) was used as the excitation source. A photomultiplier tube (PMT) was used as the detector. Time resolved delayed fluorescence decays in bare films and cavities follow eq. 3-4. The analytical solution of eq. 3 gives the expression of the delayed emission:

$$
I_{D F} \propto \gamma_{T T A}\left(\frac{\rho_{0} e^{-k_{T} t}}{1+\frac{\gamma_{T T A} \rho_{0}}{k_{T}}\left(1-e^{-k_{T} t}\right)}\right)^{2}
$$


We measured the time resolved emission at different temperatures. The excitation power $\left(l_{\text {ex }}\right)$ was recorded to calculate the initial density of the triplet annihilator $\left(\rho_{0}\right)$ after excitation (from Figure S13). Cavities absorb less light due to the strongly reflecting top mirror, and the initial triplet densities was calibrated using the cavity reflectance spectrum (Figure 2a). ${ }^{31}$ We measured the time resolved emission under 3 different excitation powers at every temperature. The kinetic parameters $\left(\nu_{T T A}\right.$ and $\left.k_{T}\right)$ were calculated by global fitting from the 3 measurements.

Transient absorption spectroscopy. Transient absorption spectra of solution and the bare film were recorded on an Edinburgh Instruments LP 980 transient absorption spectrometer with a $\mathrm{Nd}$ :YAG laser coupled to a OPO as the excitation source under $\mathrm{N}_{2}$ atmosphere.

Modeling $V_{\text {TTA }}$ in the bare film. TTA involves the diffusion and subsequent annihilation reaction. Exciton to exciton TTA of $\mathrm{DPP}(\mathrm{PhCl})_{2}$ is annihilation controlled, since the annihilation reaction is endothermic. The overall rate constant of exciton to exciton TTA then follows the Arrhenius equation:

$$
\gamma_{T T A}=A e^{\frac{-\Delta E}{k_{B} T}}(e q .11)
$$

where $A$ is a pre-exponential factor, $\Delta E$ is the energy difference between two times the $T_{1}$ energy and the $\mathrm{S}_{1}$ energy, $k_{B}$ is the Boltzmann constant, and $T$ is the absolute temperature.

Modeling $\mathrm{Y}_{\text {TTA }}$ in cavities. Exciton to polariton TTA involves the diffusion to form the excitonic triplet pair, followed by direct annihilation to the polariton. TTA in Cav3 is exothermic, and is thus diffusion controlled. The overall rate constant of exciton to polariton TTA then follow the Smolochowski equation: 


$$
\gamma_{T T A}=4 \pi r D \quad(e q .12)
$$

where $r$ is the TTA interaction radius, which correlates to the annihilation strength. The diffusion coefficient of Frenkel excitons are affected by temperature, and can be described in the following equation:

$$
D=D_{0} e^{\frac{-E_{a}}{k_{B} T}}(e q .13)
$$

where $D_{0}$ is the maximal diffusion coefficient, and $E_{A}$ is the activation energy. To model $\gamma_{\pi A A}$ in Cav3 the sum of equation 11 (with the parameters obtained from the bare film) and 12 was used.

\section{Data availability}

All characterization data and pertinent experimental procedures are provided in this article or its Supplementary Information. Additional data are also available from the corresponding author on reasonable request.

\section{Acknowledgements}

We gratefully acknowledge financial support from the European Research council (ERC2017-StG-757733) and the Knut and Alice Wallenberg Foundation (KAW 2017.0192). M.K. acknowledges funding from the European Research Council (ERC) under the European Union's Horizon 2020 research and innovation programme (grant agreement No. 852286).

\section{Author contributions}

K.B. conceived the project and supervised the work. S.M. carried out the synthesis of the annihilator molecules. C.Y. and S.M. prepared the solid film and cavity samples. C.Y. performed the spectroscopy measurements, analyzed spectroscopic data and carried out the 
corresponding simulations. M.K. provided the analysis of spin states. All authors contributed to the writing and editing of the manuscript and have given approval to its final version.

\section{Competing interests}

There are no competing interests to declare.

\section{Additional information}

Supplementary information is available for this paper at https://doi.xxx. Correspondence and requests for materials should be addressed to K.B.

\section{References}

1 Hertzog, M., Wang, M., Mony, J. \& Borjesson, K. Strong light-matter interactions: a new direction within chemistry. Chem Soc Rev 48, 937-961, doi:10.1039/c8cs00193f (2019).

2 Paravicini-Bagliani, G. L. et al. Magneto-transport controlled by Landau polariton states. Nature Physics 15, 186-190, doi:10.1038/s41567-018-0346-y (2018).

3 Frisk Kockum, A., Miranowicz, A., De Liberato, S., Savasta, S. \& Nori, F. Ultrastrong coupling between light and matter. Nature Reviews Physics 1, 19-40, doi:10.1038/s42254-018-0006-2 (2019).

4 Baranov, D. G., Wersäll, M., Cuadra, J., Antosiewicz, T. J. \& Shegai, T. Novel Nanostructures and Materials for Strong Light-Matter Interactions. ACS Photonics 5, 24-42, doi:10.1021/acsphotonics.7b00674 (2017).

5 Schneider, C., Glazov, M. M., Korn, T., Hofling, S. \& Urbaszek, B. Two-dimensional semiconductors in the regime of strong light-matter coupling. Nat. Commun. 9, 2695, doi:10.1038/s41467-018-04866-6 (2018).

6 Lu, T. C. et al. Room temperature current injection polariton light emitting diode with a hybrid microcavity. Nano Lett. 11, 2791-2795, doi:10.1021/nl2011164 (2011).

7 Ribeiro, R. F., Martínez-Martínez, L. A., Du, M., Campos-Gonzalez-Angulo, J. \& Yuen-Zhou, J. Polariton chemistry: controlling molecular dynamics with optical cavities. Chem. Sci. 9, 63256339, doi:10.1039/C8SC01043A (2018).

8 Knuppel, P. et al. Nonlinear optics in the fractional quantum Hall regime. Nature 572, 91-94, doi:10.1038/s41586-019-1356-3 (2019).

9 Zasedatelev, A. V. et al. A room-temperature organic polariton transistor. Nat. Photonics 13, 378-383, doi:10.1038/s41566-019-0392-8 (2019).

10 Thomas, A. et al. Tilting a ground-state reactivity landscape by vibrational strong coupling. Science 363, 615-619, doi:10.1126/science.aau7742 (2019).

11 Amo, A. et al. Exciton-polariton spin switches. Nat. Photonics 4, 361-366, doi:10.1038/nphoton.2010.79 (2010).

12 Coffinet, J. P. \& De Martini, F. Coherent Excitation of Polaritons in Gallium Phosphide. Phys. Rev. Lett. 22, 60-64, doi:10.1103/PhysRevLett.22.60 (1969). 
Guillaume, C. B. a. I., Bonnot, A. \& Debever, J. M. Luminescence from Polaritons. Phys. Rev. Lett. 24, 1235-1238, doi:10.1103/PhysRevLett.24.1235 (1970).

14 Du, W., Zhang, S., Zhang, Q. \& Liu, X. Recent Progress of Strong Exciton-Photon Coupling in Lead Halide Perovskites. Adv. Mater. 31, e1804894, doi:10.1002/adma.201804894 (2019).

15 Chikkaraddy, R. et al. Single-molecule strong coupling at room temperature in plasmonic nanocavities. Nature 535, 127-130, doi:10.1038/nature17974 (2016).

16 Sanvitto, D. \& Kéna-Cohen, S. The road towards polaritonic devices. Nat. Mater. 15, 10611073, doi:10.1038/nmat4668 (2016).

17 Munkhbat, B., Wersall, M., Baranov, D. G., Antosiewicz, T. J. \& Shegai, T. Suppression of photooxidation of organic chromophores by strong coupling to plasmonic nanoantennas. Sci Adv 4, eaas9552, doi:10.1126/sciadv.aas9552 (2018).

Coles, D. M. et al. Polariton-mediated energy transfer between organic dyes in a strongly coupled optical microcavity. Nat. Mater. 13, 712-719, doi:10.1038/nmat3950 (2014).

Zhong, X. et al. Energy Transfer between Spatially Separated Entangled Molecules. Angew. Chem. Int. Ed. 56, 9034-9038, doi:10.1002/anie.201703539 (2017).

20 Stranius, K., Hertzog, M. \& Börjesson, K. Selective manipulation of electronically excited states through strong light-matter interactions. Nat. Commun. 9, 2273, doi:10.1038/s41467-01804736-1 (2018).

21 Eizner, E., Martinez-Martinez, L. A., Yuen-Zhou, J. \& Kena-Cohen, S. Inverting singlet and triplet excited states using strong light-matter coupling. Sci Adv 5, eaax4482, doi:10.1126/sciadv.aax4482 (2019).

22 Berghuis, A. M. et al. Enhanced Delayed Fluorescence in Tetracene Crystals by Strong LightMatter Coupling. Adv. Funct. Mater. 29, doi:10.1002/adfm.201901317 (2019).

23 Polak, D. et al. Manipulating molecules with strong coupling: harvesting triplet excitons in organic exciton microcavities. Chem. Sci. 11, 343-354, doi:10.1039/C9SC04950A (2020).

24 Wen, S. et al. Future and challenges for hybrid upconversion nanosystems. Nat. Photonics 13, 828-838, doi:10.1038/s41566-019-0528-x (2019).

25 Ravetz, B. D. et al. Photoredox catalysis using infrared light via triplet fusion upconversion. Nature 565, 343-346, doi:10.1038/s41586-018-0835-2 (2019).

26 Gray, V., Moth-Poulsen, K., Albinsson, B. \& Abrahamsson, M. Towards efficient solid-state triplet-triplet annihilation based photon upconversion: Supramolecular, macromolecular and self-assembled systems. Coord. Chem. Rev. 362, 54-71, doi:10.1016/j.ccr.2018.02.011 (2018).

27 Ye, C., Gray, V., Mårtensson, J. \& Börjesson, K. Annihilation Versus Excimer Formation by the Triplet Pair in Triplet-Triplet Annihilation Photon Upconversion. J. Am. Chem. Soc. 141, 95789584, doi:10.1021/jacs.9b02302 (2019).

28 Pun, A. B., Campos, L. M. \& Congreve, D. N. Tunable Emission from Triplet Fusion Upconversion in Diketopyrrolopyrroles. J. Am. Chem. Soc. 141, 3777-3781, doi:10.1021/jacs.8b11796 (2019). Turro, N. J., Ramamurthy, V. \& V. Ramamurthy, J. C. S. Principles of Molecular Photochemistry: An Introduction. (University Science Books, 2009).

30 Hopfield, J. J. Theory of the Contribution of Excitons to the Complex Dielectric Constant of Crystals. Physical Review 112, 1555-1567, doi:10.1103/PhysRev.112.1555 (1958).

31 Virgili, T. et al. Ultrafast polariton relaxation dynamics in an organic semiconductor microcavity. Phys. Rev. B 83, 245309, doi:10.1103/PhysRevB.83.245309 (2011).

32 Mikhnenko, O. V., Blom, P. W. M. \& Nguyen, T.-Q. Exciton diffusion in organic semiconductors. Energy Environ. Sci. 8, 1867-1888, doi:10.1039/c5ee00925a (2015).

33 Wan, Y. et al. Cooperative singlet and triplet exciton transport in tetracene crystals visualized by ultrafast microscopy. Nat. Chem. 7, 785-792, doi:10.1038/nchem.2348 (2015).

34 Keizer, J. Diffusion effects on rapid bimolecular chemical reactions. Chem. Rev. 87, 167-180, doi:10.1021/cr00077a009 (1987).

35 Di, D. et al. Efficient Triplet Exciton Fusion in Molecularly Doped Polymer Light-Emitting Diodes. Adv. Mater. 29, 1605987, doi:doi:10.1002/adma.201605987 (2017). 
36 Ye, C. et al. Optimizing photon upconversion by decoupling excimer formation and triplet triplet annihilation. Phys. Chem. Chem. Phys. 22, 1715-1720, doi:10.1039/C9CP06561J (2020).

37 Saltiel, J., March, G. R., Smothers, W. K., Stout, S. A. \& Charlton, J. L. Spin-statistical factor in the triplet-triplet annihilation of anthracene triplets. J. Am. Chem. Soc. 103, 7159-7164, doi:10.1021/ja00414a020 (1981). 
Entry for the table of contents

Direct Evidence of a Transition from Triplet Excitons to Hybrid Light-Matter States via Triplet-Triplet Annihilation

Chen Ye, Suman Mallick, Markus Kowalewski and Karl Börjesson*

Graphical abstract

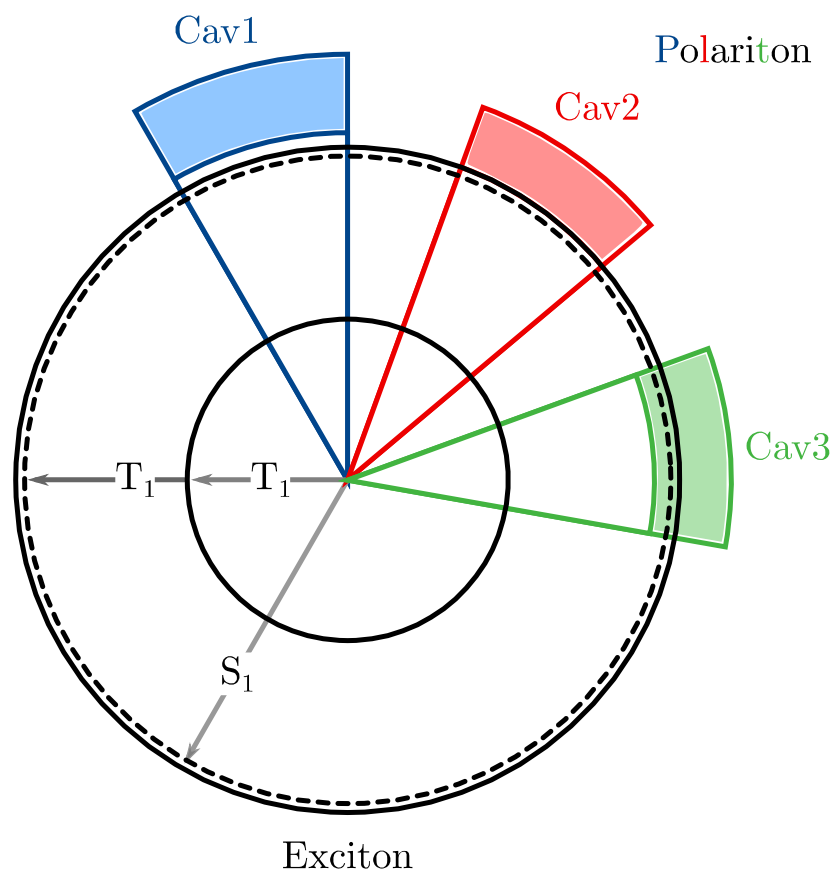

